\title{
Micro-Displacement Sensor Combined With a Fiber Ring Interrogated by an Optical Time-Domain Reflectometer
}

\author{
Mikel Bravo, José Manuel Baptista, José Luis Santos, Manuel López-Amo, Senior Member, IEEE, \\ and Orlando Frazão
}

\begin{abstract}
A micro-displacement sensing head based on two aligned cleaved fibers inserted into an optical fiber ring and interrogated by an optical time-domain reflectometry is presented. The sensor configuration is characterized for measuring both longitudinal and axial micro-displacements, showing a sensitivity of 0.07 and $2.67 \mathrm{~dB} / \mu \mathrm{m}$ for longitudinal and axial microdisplacements, respectively. A multiplexing system using two of these configurations is also studied.
\end{abstract}

Index Terms-Fiber ring, air-gap, OTDR, displacement sensor.

\section{INTRODUCTION}

$\mathbf{O}$ TDR (optical time-domain reflectometry) monitoring systems for communication networks have been also extensively used to interrogate fiber optic sensors. Different intensity sensor systems have been demonstrated using OTDR interrogation set-ups, such as local or remote sensor interrogation systems or multiplexed systems [1]-[4]. Also, OTDR systems have been employed to characterize ring structures [5]. Ring structures have been previously used in single sensor systems and for optical sensor multiplexing systems [6]. In the same way, C. Wang analyzed these structures extensively in one of his works [7], which summarizes some kind of rings sensing heads, including the utilization of an air-gap sensor inside the loop for gas measurement. These fiber rings are also called "fiber loop ring down" in his review and in other works these structures were developed for pressure [8], temperature [9], strain [10], refractive index [11] sensor systems. These

Manuscript received March 28, 2013; accepted October 25, 2013. Date of publication November 5, 2013; date of current version January 10, 2014 This work was supported in part by the European Cost Action under Grant TD-1001 "OFSeSa" and in part by the Spanish Government under Project TEC2010-20224-C02-01. The associate editor coordinating the review of this paper and approving it for publication was Prof. Ravinder S. Dahiya.

M. Bravo and M. López-Amo are with the Departamento de Ingeniería Eléctrica y Electrónica, Universidad Pública de Navarra, Pamplona 31006, Spain (e-mail: mikel.bravo@unavarra.es; mla@unavarra.es).

J. M. Baptista is with INESC Porto, Porto 4169-007, Portugal, and also with the Centro de Ciências Exatas, Universidade da Madeira, Funchal 9000-390, Portugal (e-mail: jmb@inescporto.pt).

J. L. Santos is with INESC Porto, Porto 4169-007, Portugal, and also with the Faculdade de Ciências da Universidade do Porto, Porto 4169-007, Portugal (e-mail: josantos@fc.up.pt).

O. Frazão is with INESC Porto, Porto 4169-007, Portugal (e-mail: ofrazao@inescporto.pt).

Color versions of one or more of the figures in this paper are available online at http://ieeexplore.ieee.org.

Digital Object Identifier 10.1109/JSEN.2013.2288596

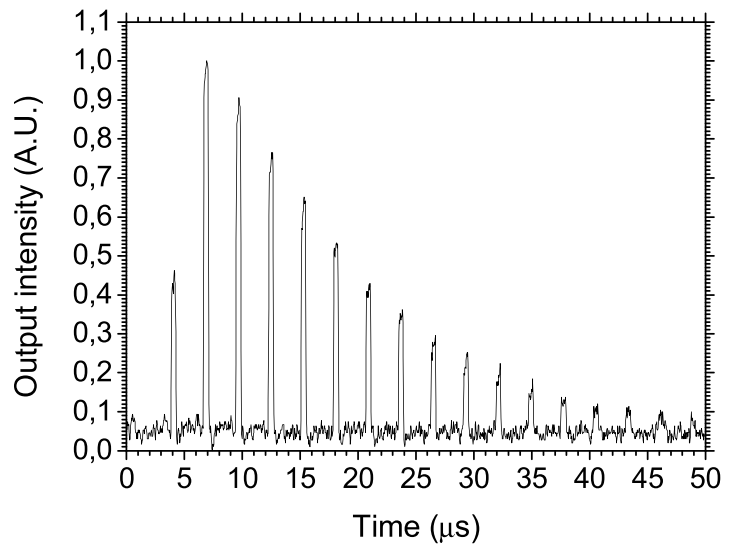

Fig. 1. Time response of a classical fiber ring-down setup.

systems usually use a modulated source and a photodetector to measure the different received light magnitudes. In these published works, the authors proposed a setup in which an optical pulse is coupled into a fiber loop. This pulse circulates around the ring down fiber loop and a part of the power which is circulating is recovered and detected by a photodetector which offers a train of pulses, similar to the train we have replicated in figure 1 . The result was achieved measuring a fiber ring setup formed by a variable coupler in a fiber ring structure, and using a laser source modulated by an electrooptical modulator (EOM) at one of the input ports, and a photodetector at one of the output ports.

The separation between each pulse corresponds to the time which the light spends inside the ring. This time is proportional to the ring cavity length and the light velocity inside the fiber, and is described as follows:

$$
T=D \frac{n}{c}
$$

where $T$ is the time between each peak, $D$ is the cavity length, $c$ is the speed of light in vacuum and $n$ is the refraction index of the fiber.

On the other hand, air gaps between two standard fibers have been extensively used before [12]. Some authors call this structure as etalon, extrinsic Fabry-Perot interferometer or air gap sensor. One of the most developed sensors was made for strain measurements [13]-[15]. These air cavities could behave as Fabry-Perot interferometers or/and as variable attenuators. 


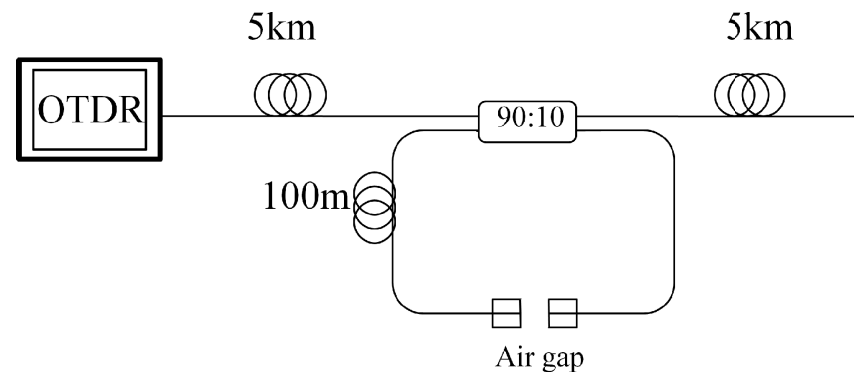

Fig. 2. Experimental setup of the fiber ring resonator combined with the proposed sensing head monitored by an OTDR.

When the air cavity sensor head is placed inside an optical fiber ring, part of the optical power inside the ring structure recirculates, and the multiple reflections produced by the structure allows a clear measurement and identification of the sensor reflections when multiplexed into a fiber network.

Nowadays, the scientific community has made a lot effort on developing fiber optic sensors based on wavelength encoded, distributed procedures, Rayleigh backscattering processing, and so on. This fact has diverted the attention of the intensity sensors which are very attractive for many commercial applications because of their simplicity and, principally, their costs.

This work presents a alternative sensing configuration that includes an air cavity between two cleaved fibers inserted into a fiber optic ring which is interrogated by a commercial OTDR. The arrangement presents good operational characteristics in a simple configuration for micro-displacement sensing, principally, improving the dynamic range. This configuration is also characterized in a multiplexing arrangement of two sensors in series.

\section{SETUP}

This work is organized in two independent parts: firstly, sensing head development and characterization; and secondly, sensor multiplexing experimental demonstration.

The proposed sensing head (figure 2) is based on a fiber ring structure. An air cavity, which is formed by two cleaved and aligned SMF28 fibers, is placed inside the ring. This ring is composed by a 90:10 low insertion loss optical coupler. Finally, this structure is interrogated by an OTDR. The sensor is placed between two $5 \mathrm{~km}$ fiber spools.

Unlike time response of the fiber loop ring-down setup presented in the introduction, the OTDR measures the backscattered and reflected signal generated by an optical pulse launched by itself at the beginning. Because of that, the response of a fiber optic ring is different when the OTDR analyzes it. The figure 3 shows two different traces: The thickest one belongs to the OTDR response of the structure presented in figure 2 without the air gap cavity; on the contrary, the second trace displays the OTDR response for figure 2 structure, the comprehends the fiber ring with the cavity. Both of them are explained thoroughly below.

In first case, the thickest trace, it is perceived a small power increment due to the contribution of the Rayleigh produced

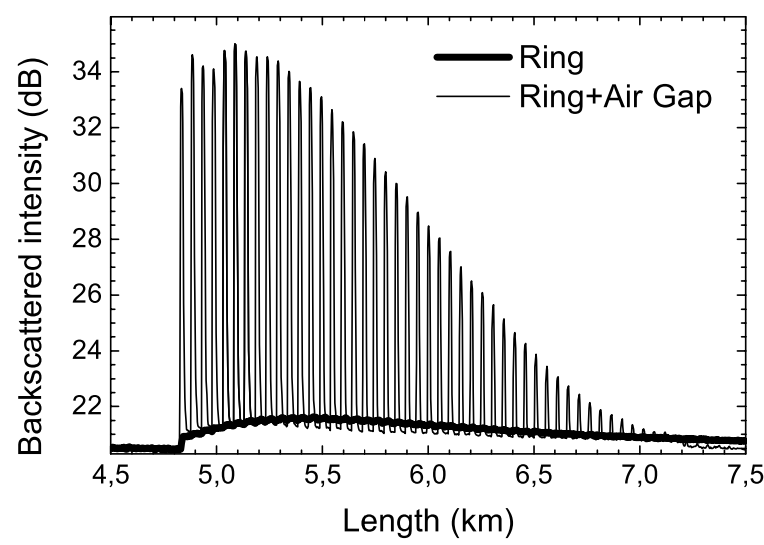

Fig. 3. OTDR trace of the proposed configuration.

into the ring and the $5 \mathrm{~km}$ spool connected after the sensor structure. Therefore, when the OTDR pulse reaches for first time the sensor, part of its power is divided in an unevenly way (90:10). 90 percent of this pulse goes to the $5 \mathrm{~km}$ spool, located after the coupler, generating some Rayleigh scattering which is added to the Rayleigh produced by the $10 \%$ pulse power which enters into the $100 \mathrm{~m}$ of the ring fiber length. When the pulse that travels through the ring reaches the coupler again, part of its power (10\%) is coupled to the $5 \mathrm{~km}$ spool illuminating again the fiber and the rest of its power (90\%) is coupled back to the ring until there is pulse power enough to travel inside the ring.

The second trace is taken when the air-gap cavity is placed inside the ring thus $\sim 4 \%$ of light is reflected in each facet of the air cavity. The plot shows a main behaviour similar to the ring without the cavity, but it has superimposed each $\sim 4 \%$ peaks reflections created in every spin.

It must be highlighted that the peaks also offer information of the ring length. The OTDR measured distance between two peaks is about $50 \mathrm{~m}$ when $\sim 100 \mathrm{~m}$ is the real length; this is due to the OTDR principle of operation. The OTDR calculates each point power position with the following equation.

$$
\text { Distance }=\frac{c \tau}{2 n} \text {. }
$$

Thus, one event generated in a point of the fiber reaches the OTDR after the light arrives at this event and goes back to the OTDR. In this situation, when the pulse arrives to the ring, each peak is created every $\sim 50 \mu$ s which is the time that it takes for the optical pulse to complete a spin. This time value has been obtained by using equation 1 . To calculate the distance that corresponds to the time interval between consecutive peaks, this data is replaced in the previous equation and the solution, as it is measured with the OTDR, is $\sim 50 \mathrm{~m}$.

Therefore, when the air-gap length changes an increment of the losses in the ring is discernible. In fact, figure 4 depicts the peaks evolution while the cavity length changes. As is explained above, the more loss into the cavity, the less time the pulse is spinning inside the ring. Hence, this information can be used for micro-displacement measurement purposes.

At this stage, the next issue was to study how these sensors can be multiplexed. Different techniques for sensor 


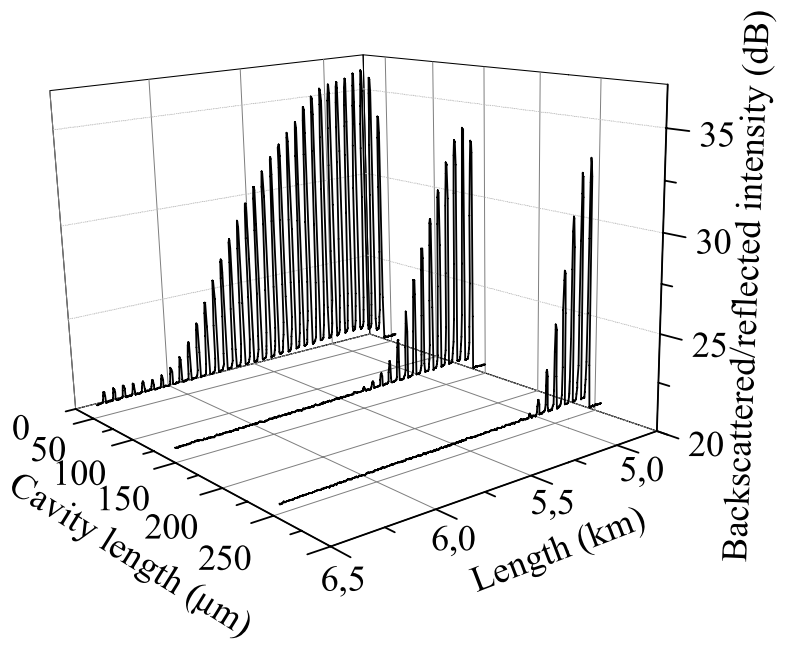

Fig. 4. OTDR trace evolution as displacement increases.

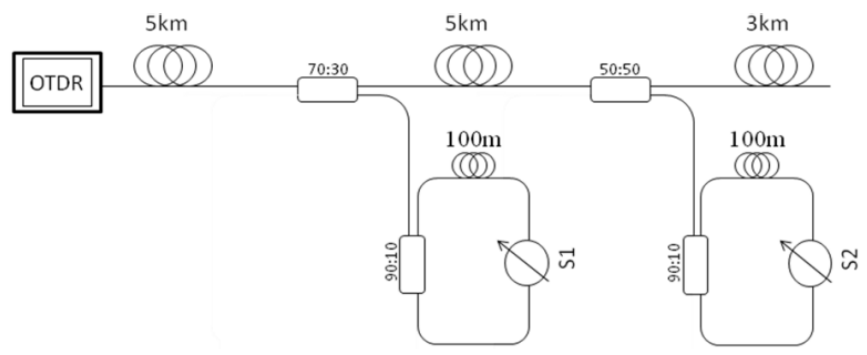

Fig. 5. Sensors multiplexing experimental setup.

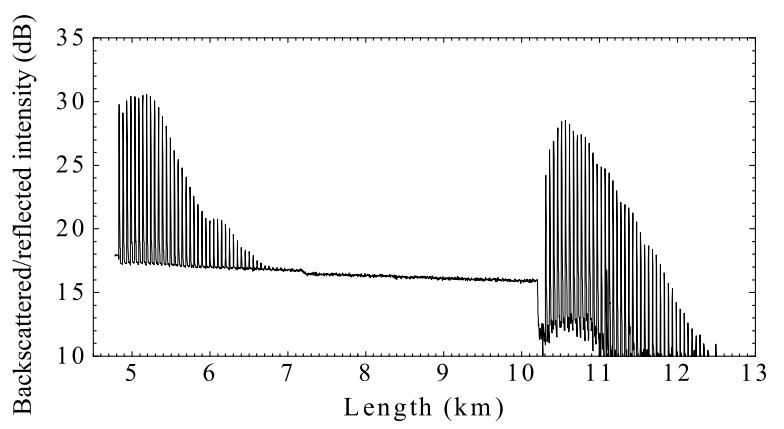

Fig. 6. OTDR trace of the two sensors multiplexing set-up.

multiplexing have been proposed using the OTDR as interrogation device [1]-[2]. Figure 5 shows the proposed structure. A tree configuration has been selected in order to avoid the crosstalk between sensors. The sensors have been added to the main optical path by optical couplers which have been separated by different fiber spools. Thus, in general the spools simulate a real network and each of them has different objectives: the first one avoids the death zone of the OTDR; the second spool prevents the sensors response to be superimposed and the last one creates a Rayleigh threshold in order to keep the signal above the detection noise.

Figure 6 presents a complete OTDR measured trace for both sensors. The plot depicts both sensors' peak response separated as expected. The second sensor's response starts about $3 \mathrm{~dB}$ lower from the Rayleigh threshold due to the 50:50 coupler used for multiplexing it.

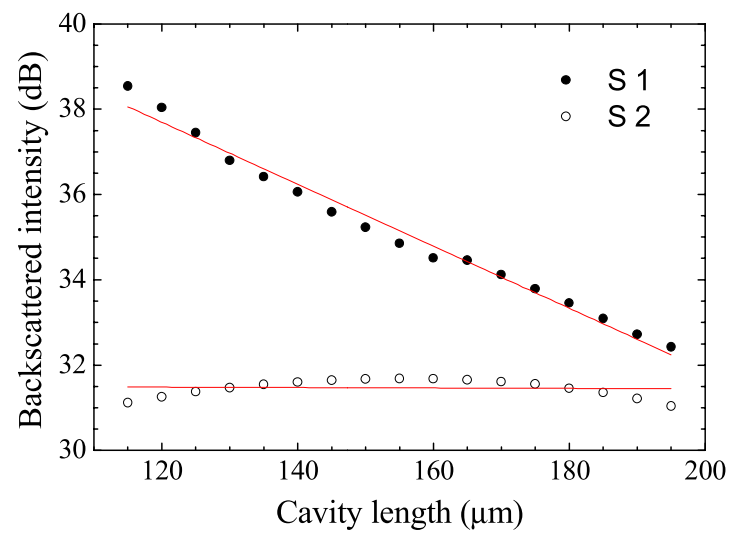

Fig. 7. Proposed sensor longitudinal displacement behavior in a multiplexed network of two sensors.

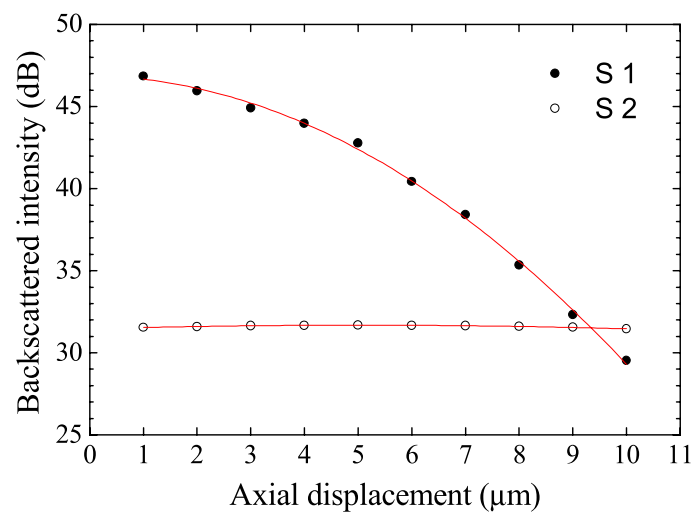

Fig. 8. Received power from sensor (S1) behavior when an axial lateral displacement is induced.

\section{RESUlts}

Two sensors based on an air-gap have been multiplexed and interrogated by an OTDR. The results have been performed with two micrometer translation stages in which the two cleaved fibers are placed one in front of the other creating the air-gap cavity. Both translation stages have a precision of $1 \mu \mathrm{m}$ per step.

Figure 7 shows the behavior of one sensor when longitudinal displacement is applied and the behavior of the second one when it is not stressed. The displacement measurement from the first cavity length (S1) was obtained by averaging the values of the fifth, sixth and seventh peaks of the pulse train acquired by the OTDR, trace shown in figure 3 . These peaks had been selected after a study which analyzed each peak behavior. After comparing all peaks contributions, this study established which peaks showed the best characteristics to be used, principally the best SNR. The experiment consisted in increasing the $\mathrm{S} 1$ cavity length by $5 \mu \mathrm{m}$ steps. The results were taken for a $115 \mu \mathrm{m}$ initial cavity length and showed a quasi linear behavior in the $80 \mu \mathrm{m}$ measurement range. A sensitivity of $0.07 \mathrm{~dB} / \mu \mathrm{m}$ was obtained with a dynamic range of $\sim 6 \mathrm{~dB}$. It was observed some variation of the intensity returned from sensor $2(\sim 1 \mathrm{~dB})$.

A cavity axial misalignment test was also carried out, obtaining the results presented in Figure 8. 
For this experiment, $40 \mu \mathrm{m}$ initial cavity length was used and steps of $1 \mu \mathrm{m}$ axial lateral displacements were applied. A linear behavior was obtained for the first $5 \mu \mathrm{m}$ and after that, the received light intensity, as expected, dropped abruptly. For the first $5 \mu \mathrm{m}$ measurement range, the misalignment sensor showed a sensitivity of $2.67 \mathrm{~dB} / \mu \mathrm{m}$. In this case the variations of sensor 2 output were observed to be negligible.

Intensity based sensors suffer from the problem of system optical power fluctuations not related with those induced by the measurand changes. Therefore, this is also a drawback of the proposed configuration, that can be overcame by the consideration of an adequate signal referencing approach, a topic that is currently under study.

\section{CONCLUSION}

An air-gap based on micro-displacement sensing head inserted in a fiber ring and interrogated by an OTDR has been experimentally implemented and characterized. A sensitivity of $0.07 \mathrm{~dB} / \mu \mathrm{m}$ using this type of sensing head was obtained for longitudinal displacement. In the same conditions, an axial lateral micro-displacement characterization was also performed presenting a sensitivity of $2.67 \mathrm{~dB} / \mu \mathrm{m}$. The combination of an air cavity and a fiber ring resonator in the way described in this work, proved to be an interesting sensing approach. It shows intrinsic compatibility with time division multiplexing, demonstrating the viability of the development of extended networks multiplexing a higher number of this type of sensors.

\section{REFERENCES}

[1] M. Bravo, M. Fernandez-Vallejo, and M. Lopez-Amo, "Hybrid OTDRfiber laser system for remote sensor multiplexing," IEEE Sensors J., vol. 12, no. 1, pp. 174-178, Jan. 2012.

[2] N. M. P. Pinto, O. Frazao, J. M. Baptista, and J. L. Santos, "Quasidistributed displacement sensor for structural monitoring using a commercial OTDR," Opt. Lasers Eng., vol. 44, no. 8, pp. 771-778, 2006.

[3] M. Bravo, J. Sáenz, M. Bravo-Navas, and M. López-Amo, "Concrete beam bending test monitorization using a high strain fiber optic sensor," J. Lightw. Technol., vol. 30, no. 8, pp. 1085-1089, Apr. 15, 2012.

[4] M. Bravo, J. M. Baptista, J. L. Santos, M. López-Amo, and O. Frazão, "Ultralong $250 \mathrm{~km}$ remote sensor system based on a fiber loop mirror interrogated by an optical time-domain reflectometer," Opt. Lett., vol. 36, no. 20, pp. 4059-4061, 2011.

[5] A. Küng, J. Budin, L. Thévenaz, and P. A. Robert, "Optical fiber ring resonator characterization by optical time-domain reflectometry," Opt. Lett., vol. 22, no. 2, pp. 90-92, 1997.

[6] R. Fernández de Caleya, M. López-Amo, and J. A. Martín-Pereda, "Low cost time division multiplexing of identical optical fibre ring intensity sensors," Int. J. Optoelectron., vol. 9, no. 1, pp. 81-88, 1994.

[7] C. Wang, "Fiber loop ringdown-A time-domain sensing technique for multi-function fiber optic sensor platforms: Current status and design perspectives," Sensors, vol. 9, no. 10, pp. 7595-7621, 2009.

[8] C. Wang and S. T. Scherrer, "Fiber ringdown pressure sensors," Opt. Lett., vol. 29, no. 4, pp. 352-354, 2007.

[9] C. Wang, "Fiber ringdown temperature sensors," Opt Eng., vol. 44, no. 3, pp. 030503-1-030503-2, 2005.

[10] N. Ni, C. C. Chan, X. Y. Dong, J. Sun, and P. Shum, "Cavity ring-down long-period fibre grating strain sensor," Meas. Sci. Technol., vol. 18, no. 10 , pp. 3135-3138, 2007

[11] N. Ni, C. C. Chan, L. Xia, and P. Shum, "Fiber cavity ring-down reflactive index sensor," IEEE Photon. Technol. Lett., vol. 20, no. 16, pp. 1351-1353, Aug. 15, 2008.

[12] Y.-J. Rao, "Recent progress in fiber-optic extrinsic Fabry-Perot interferometric sensors," Opt. Fiber Technol., vol. 12, no. 3, pp. 227-237, 2006.
[13] J. Sirkis, T. A. Berkoff, R. T. Jones, H. Singh, A. D. Kersey, E. J. Friebele, et al., "In-line fiber etalon (ILFE) fiber-optic strain sensors," J. Lightw. Technol., vol. 13, no. 7, pp. 1256-1263, Jul. 1995.

[14] Y.-L. Lo, J. S. Sirkis, and C.-C. Chang, "Passive signal processing of inline fiber etalon sensors for high strain-rate loading," J. Lightw. Technol., vol. 15, no. 8, pp. 1578-1586, Aug. 1997.

[15] L. Liu and G. F. Fernando, "A frecuency division multiplexed lowfinesse fiber optic Fabry-Perot sensor system for strain an displacement measurements," Rev. Sci. Instrum., vol. 71, no. 3, pp. 1275-1278, 2000.

Mikel Bravo was born in Deba, Guipuzcoa, Spain, in June 1983. He received the Telecommunication Technical Engineering and Communications Master degrees from the Universidad Pública de Navarra, Spain in 2007 and 2010, respectively.

In 2008, he joined the Optical Communications Group, Department of Electrical and Electronic Engineering, Universidad Pública de Navarra.

His research interests are in structural health monitoring, fiber Bragg gratings, intensity bending sensors, multiplexing architectures, optical amplifiers, and optical networks.

José Manuel Baptista (M'03) graduated with a Degree in electrical and computer engineering (telecommunications and computers) from the University of Porto, Porto, Portugal, in 1991, the M.Sc. degree in physics of laser communications from the University of Essex, Colchester, U.K., in 1992, and the Ph.D. degree in electrical and computer engineering from the University of Porto, in 2002. Currently, he is an Associate Professor with the University of Madeira and a Senior Researcher in the Optoelectronics and Electronics Systems Unit at INESC Porto. He is author of more than 60 journal publications and author of two patents. His research interests are in the area of fiber-optic sensors, fiber-optic communications, and fiber-optic technologies.

José Luis Santos graduated in 1983 with a Degree in applied physics (optics and electronics) from the University of Porto, Portugal, where he received the Ph.D. degree in physics in 1993 for research on fiberoptic sensing. He is a Full Professor in the Physics Department, University of Porto, and is in charge of the Optoelectronics and Electronic Systems Unit of INESC Porto. His main research interest is optical fiber sensing. Prof. Santos is a member of the Optical Society of America (OSA) and the International Society for Optical Engineers (SPIE)

Manuel López-Amo (M'91, SM'98) was born in Madrid, Spain, in 1960 He received the M.Sc. telecommunication engineering degree and the Ph.D. degree from the Universidad Politécnica de Madrid, Spain in 1985 and 1989, respectively. From 1985 to 1996, he was with the Photonic Technology Department, Universidad Politécnica de Madrid, where in 1990 he became an Associate Professor.

He was a Visiting Researcher at INTEC, University of Ghent, Belgium. and at British Telecom. Research Laboratories (Ipswich, U.K.).

In 1996, he moved to the Public University of Navarra (Pamplona, Spain) where he became a Full Professor in the Electrical and Electronic Engineering Department and is currently the Head of the Optical Communications Group of this department. He has been Chairman of the Optoelectronic Committee of Spain. He has been leader of more than 20 research projects and he has coauthored more than 100 works in international refereed journals related with fiber-optic networks, optical amplifiers, fiber-optic sensors, and integrated optics. He is a member of the technical committees of the International Conference on Fiber Optic Sensors (OFS), the European Workshop on Optical Fiber Sensors (EWOFS), and European Cost TD1001 action, among others. Prof. López-Amo is senior member of the IEEE and member of the OSA.

Orlando Frazão received the Degree in physics engineering (optoelectronics and electronics) from the University of Aveiro, Portugal, and the Ph.D degree in physics from the University of Porto, Portugal, in 2009. From 1997 to 1998, he was with the Institute of Telecommunications, Aveiro. Presently, he is a Senior Researcher with the Optoelectronics and Electronic Systems Unit, INESC Porto. He has published about 250 papers, mainly in international journals and conference proceedings, and his present research interests includes optical fiber sensors and optical communications. Dr. Frazão is a member of OSA and Senior Member of SPIE. 